\title{
Dynamics of the Flow Pattern in a Baffled Mixing Vessel with an Axial Impeller
}

\author{
O. Brůha, T. Brůha, I. Fořt, M. Jahoda
}

This paper deals with the primary circulation of an agitated liquid in a flat-bottomed cylindrical stirred tank. The study is based on experiments, and the results of the experiments are followed by a theoretical evaluation. The vessel was equipped with four radial baffles and was stirred with a six pitched blade impeller pumping downwards. The experiments were concentrated on the lower part of the vessel, where the space pulsations of the primary loop, originated due to the pumping action of the impeller. This area is considered to be the birthplace of the flow macroinstabilities in the system - a phenomenon which has been studied and described by several authors. The flow was observed in a vertical plane passing through the axis of the vessel. The flow patterns of the agitated liquid were visualized by means of Al micro particles illuminated by a vertical light knife and scanned by a digital camera. The experimental conditions corresponded to the turbulent regime of agitated liquid flow.

It was found that the primary circulation loop is elliptical in shape. The main diameter of the primary loop is not constant. It increases in time and after reaching a certain value the loop disintegrates and collapses. This process is characterized by a certain periodicity and its period proved to be correlated to the occurrence of flow macroinstability. The instability of the loop can be explained by a dissipated energy balance. When the primary loop reaches the level of disintegration, the whole impeller power output is dissipated and under this condition any flow alteration requiring additional energy, even a very small vortex separation, causes the loop to collapse.

Keywords: mixing, axial impeller, primary circulation loop, oscillation, macroinstability.

\section{Introduction}

A mechanically agitated system under a turbulent regime of flow with or without internals (radial baffles, draft tubes, coils etc.) consists of a broad spectrum of eddies from the size of the main (primary) circulation loop (PCL) of the agitated batch down to the dissipative vortices corresponding to micro-scale eddies.

This study deals with an experimental and theoretical analysis of the behaviour of the flow pattern, and mainly the PCL in an agitated liquid in a system with an axial flow impeller and radial baffles. Some characteristics of the investigated behaviour are considered to be in correlation with the occurrence of flow macroinstabilities (FMIs), i.e. the flow macro-formation (vortex) appearing periodically in various parts of a stirred liquid. The flow macroinstabilities in a mechanically agitated system are large-scale variations of the mean flow that may affect the structural integrity of the vessel internals and can strongly affect both the mixing process and the measurement of turbulence in a stirred vessel. Their space and especially time scales considerably exceed those of the turbulent eddies that are a well known feature of mixing systems. The FMIs occur in a range from several up to tens of seconds in dependence on the scale of the agitated system. This low-frequency phenomenon is therefore quite different from the main frequency of an incompressible agitated liquid corresponding to the frequency of revolution of the impeller.

Generally, experimental detection of FMIs is based on frequency analysis of the oscillating signal (velocity, pressure, force) in long time series and frequency spectra, or more sophisticated procedures (proper orthogonal decomposition of the oscillating signal, the Lomb period gram or the velocity decomposition technique) are used to determine the FMI frequencies. A theoretical method for finding FMIs could contribute significantly to a deeper understanding of fluid flow behaviour in stirred vessels, e.g. a description of the circulation patterns of a agitated liquid, application of the theory of deterministic chaos, knowledge of turbulent coherent structures, etc. [1-10].

This study investigates oscillations of the primary circulation loop (the source of FMIs) in a cylindrical system with an axial flow impeller and radial baffles, aiming at a theoretical description of the hydrodynamical stability of the loop. However, no integrated theoretical study of the MI phenomenon has been presented up to now.

\section{Experimental}

The experiments were performed in a flat-bottomed cylindrical stirred tank of inner diameter $T=0.29 \mathrm{~m}$ filled with water at room temperature with the tank diameter height $H=T$. The vessel was equipped with four radial baffles (width of baffles $b=0.1 T$ ) and stirred with a six pitched blade impeller (pitch angle $45^{\circ}$, diameter $D=\mathrm{T} / 3$, width of blade $W=D / 5$ ), pumping downwards. The impeller speed was adjusted $n=400 \mathrm{rpm}=6.67 \mathrm{~s}^{-1}$ and its off-bottom clearance $C$ was $T / 3$ (see Fig. 1).

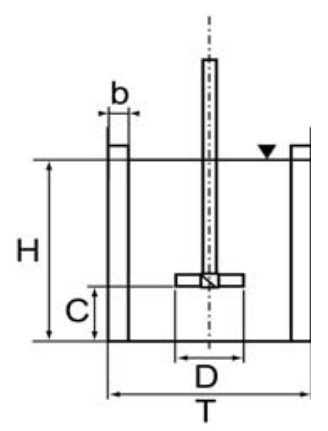

Fig. 1: Pilot plant experimental equipment 

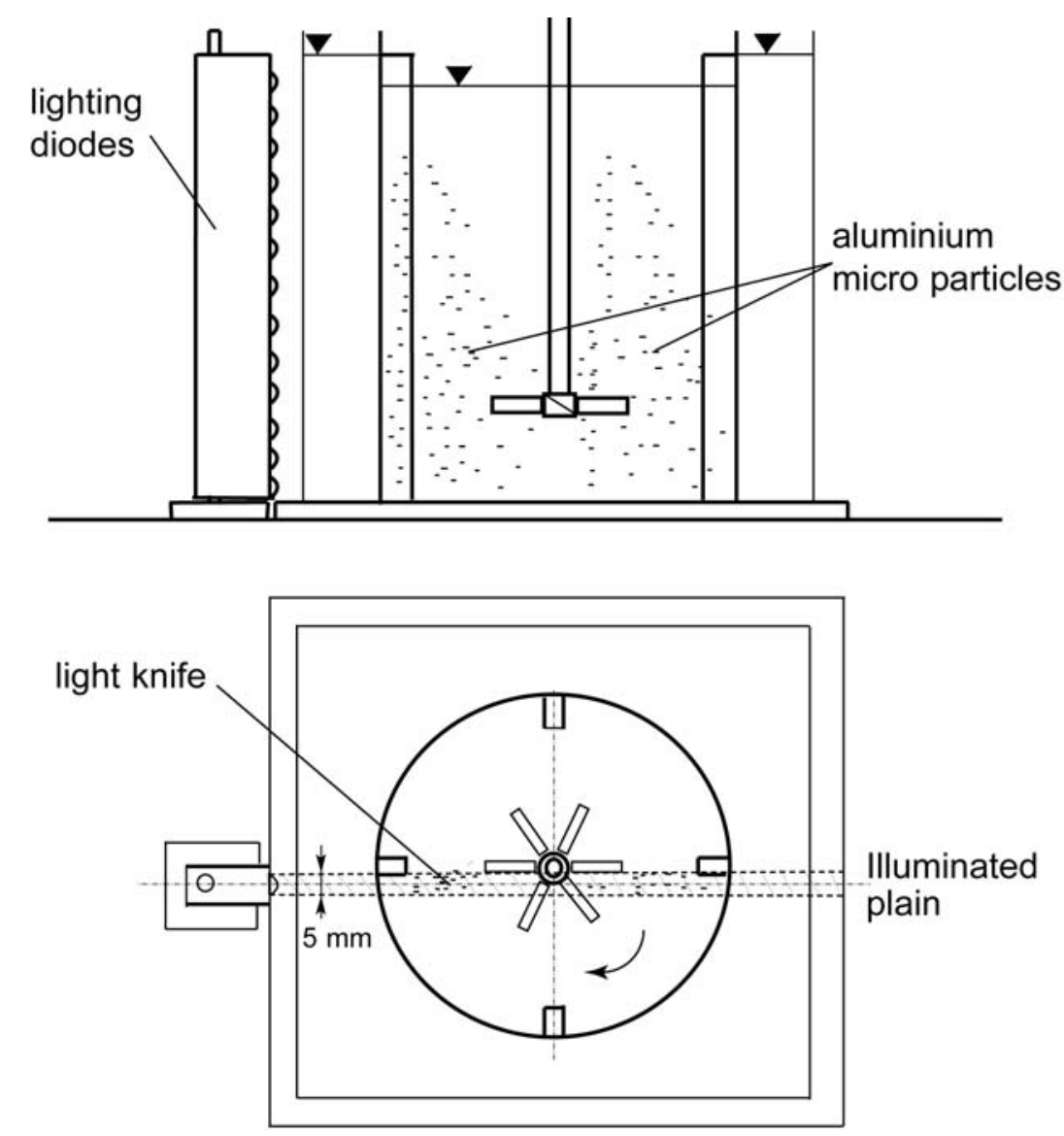

Fig. 2: Experimental technique of flow visualization

The flow under the parameters mentioned above was turbulent, with the impeller Reynolds number value $\operatorname{Re}_{\mathrm{M}}=6.22 \cdot 10^{4}$.

The flow was observed in a vertical plane passing through the vessel (vertical section of the vessel) in front of the adja-

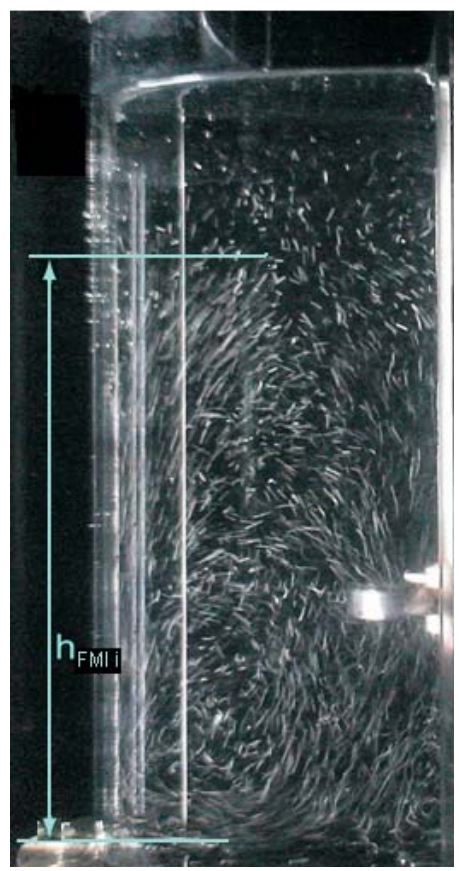

Fig. 3: Visualization of the PCL cent baffles. The flow patterns of the agitated liquid were visualized by means of $A l$ micro particles $0.05 \mathrm{~mm}$ in diameter spread in water and illuminated by a vertical light knife $5 \mathrm{~mm}$ in width, see Fig. 2.

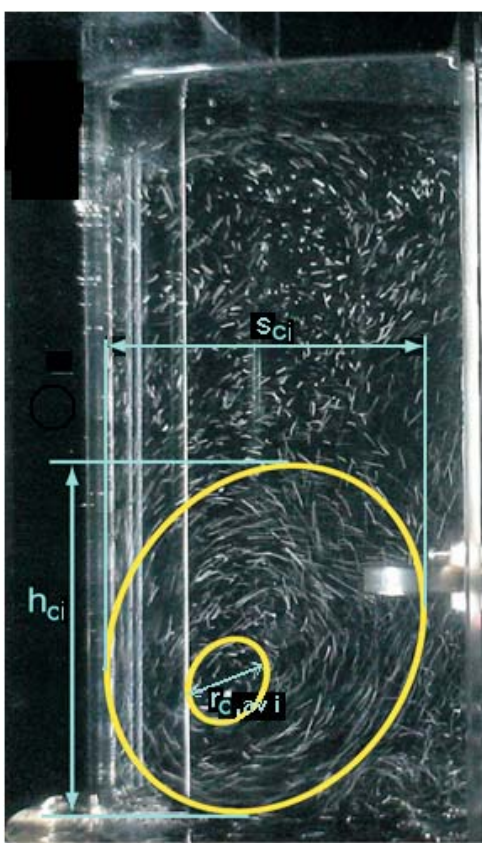

Fig. 4: Visualization of flow macro formation 


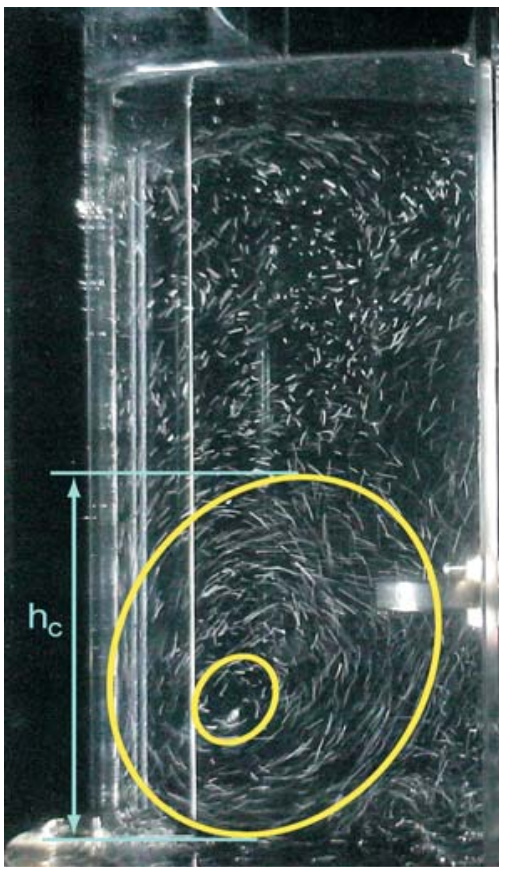

a)

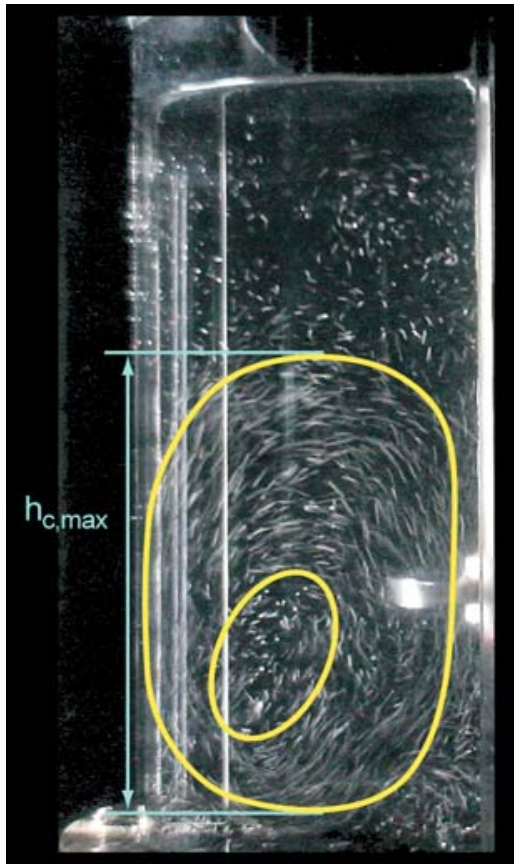

b)

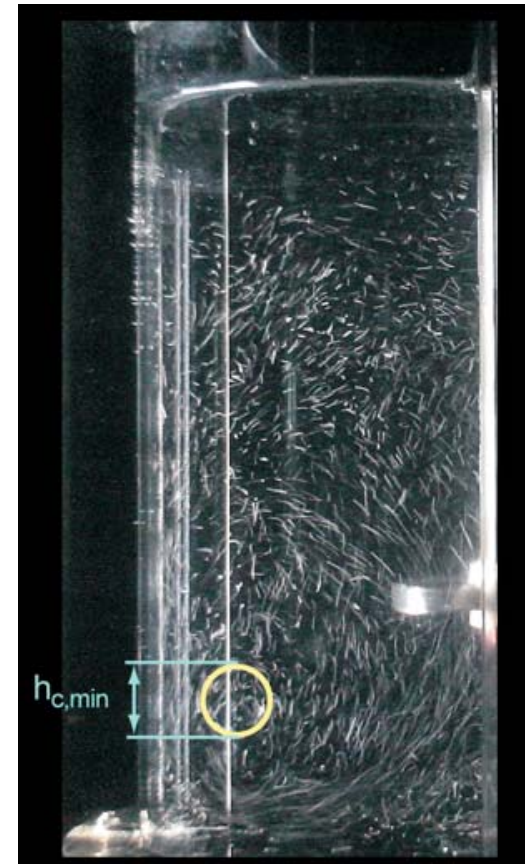

c)

Fig. 5: a) PCL growing, b) PCL at maximum height, c) PCL after its collapse

The visualized flow was scanned by a digital camera; series of shots were generated with a time step of $0.16 \mathrm{~s}$ and were analyzed by appropriate graphic software. The total length of the analyzed record was $30.24 \mathrm{~s}$. The characteristics observed and analyzed were:

a) the shape and sizes of the PCL,

b) the size of the PCL core,

c) the positions of the top of the flow macro formation and the corresponding functions were obtained:

a) the height of the PCL $h_{\mathrm{ci}}=h_{\mathrm{ci}}(t)$ (see Fig. 3),

b) the width of the PCL $s_{\mathrm{ci}}=s_{\mathrm{ci}}(t)$ (see Fig. 3),

c) the equivalent mean diameter of the PCL core $r_{\text {c,av i }}=r_{\text {c,av i }}(t)$ (see Fig. 3),

d) the height of the top of the flow macro formation $h_{\mathrm{FMI}, \mathrm{i}}=h_{\mathrm{FMI}, \mathrm{i}}(t)$ (see Fig. 4 ).

\section{Results of experiments}

The analysis of the experiments provided the following findings:

The PCL can be described as a closed stream tube with a vertical section elliptical in shape with a core. The flow in the elliptical annular area is intensive and streamlined, while the core is chaotic and has no apparent streamline characteristics. The flow in the remaining upper part is markedly steadier. This is in agreement with the earlier observations of some authors $[6,7]$.

The flow process is characterized by three stages. In the first stage, the PCL grows to a certain size (its shape can be approximated as elliptical). Then a quasi-steady stage follows, when the PCL remains at a constant size for a short time. In the next stage the PCL collapses into very small flow formations (vortices) or disintegrates into chaotic flow, see Fig. 5a-c.

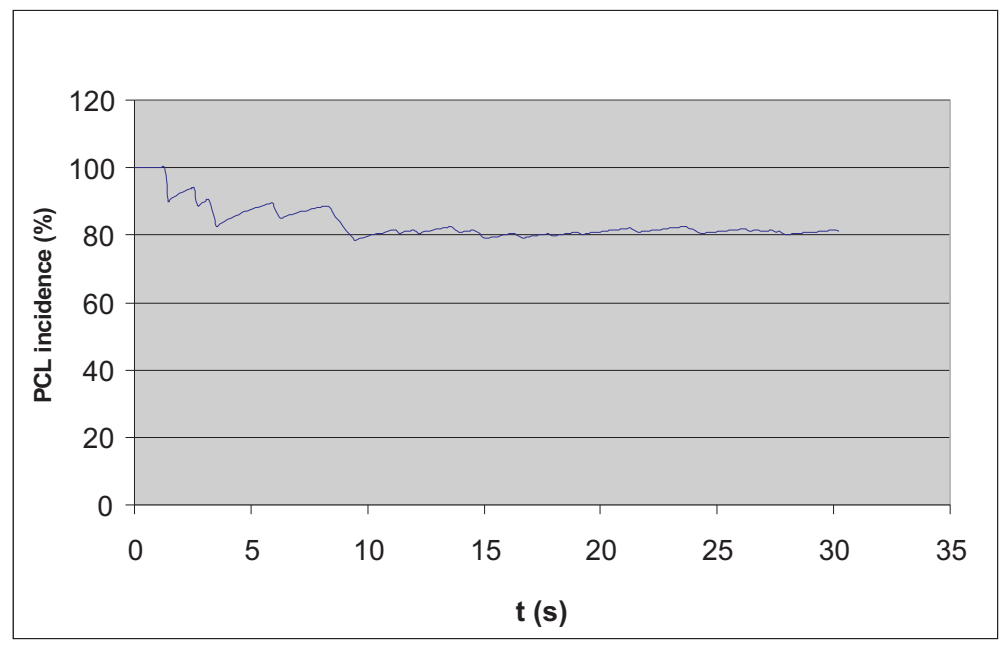

Fig. 6: Dependence of PCL incidence on time for the whole analyzed time 


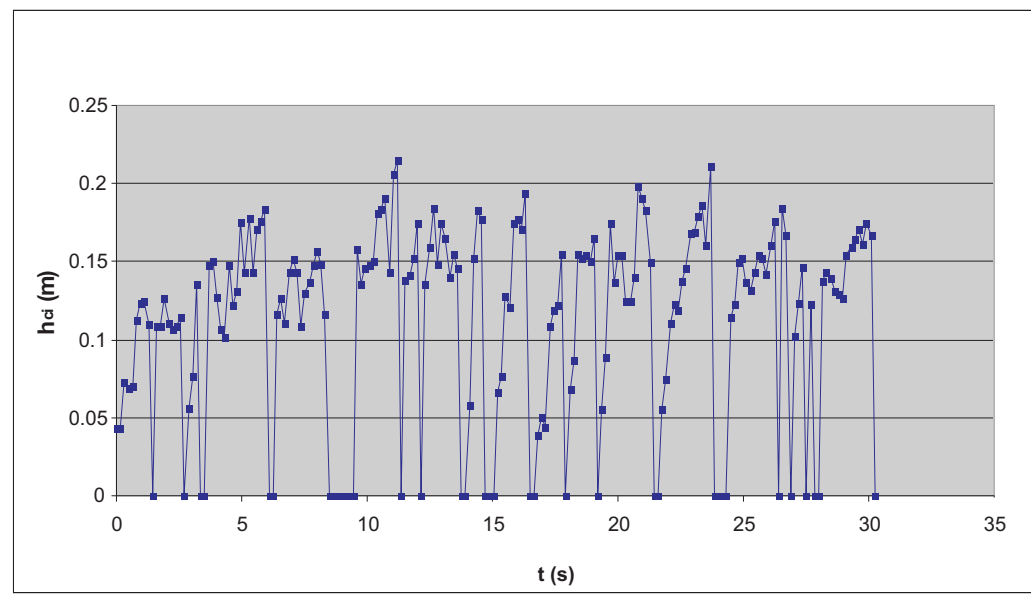

Fig. 7: Dependence of the vertical dimension of PCL $h_{\mathrm{ci}}$ on time for the whole analyzed time

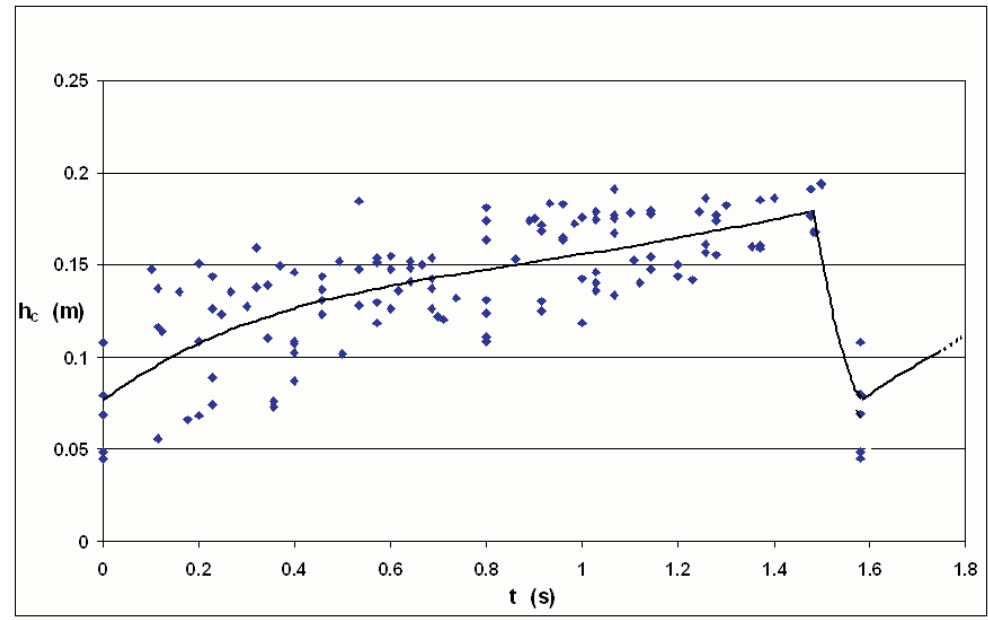

Fig. 8: Time evolution of the height of the PCL (average cycle time $t_{\mathrm{c}, \mathrm{av}}=1.59 \mathrm{~s}$, maximum height $h_{\mathrm{c}, \max }=0.181 \mathrm{~m}, h_{\mathrm{c}, \max } / H=0.62$ ) for one cycle of PCL oscillation

The process (oscillation of the PCL) is apparently characterized by a certain periodicity. The function of the incidence of the PCL in time is illustrated in Fig. 6, which shows that the PCL incidence ratio approaches $80 \%$ of the considered time.
The graph in Fig. 7 (vertical dimension of the PCL as a function of time) illustrates this process for the whole analysed time of $30.24 \mathrm{~s}$.

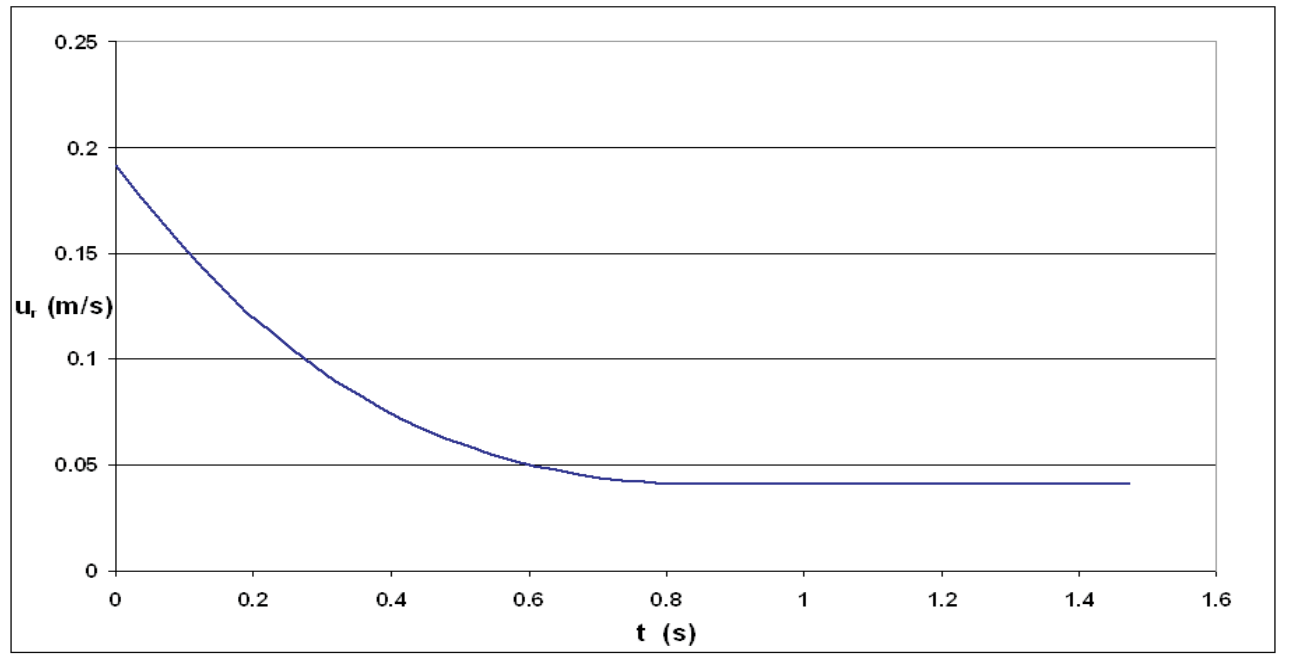

Fig. 9: Dependence of the mean rising velocity $u_{\mathrm{r}}$ of the PCL on time $\left(u_{\mathrm{r}}=\mathrm{d} h / \mathrm{d} t=0.042 \mathrm{~m} / \mathrm{s}=\right.$ const., $\left.t>0.77 \mathrm{~s}\right)$ 


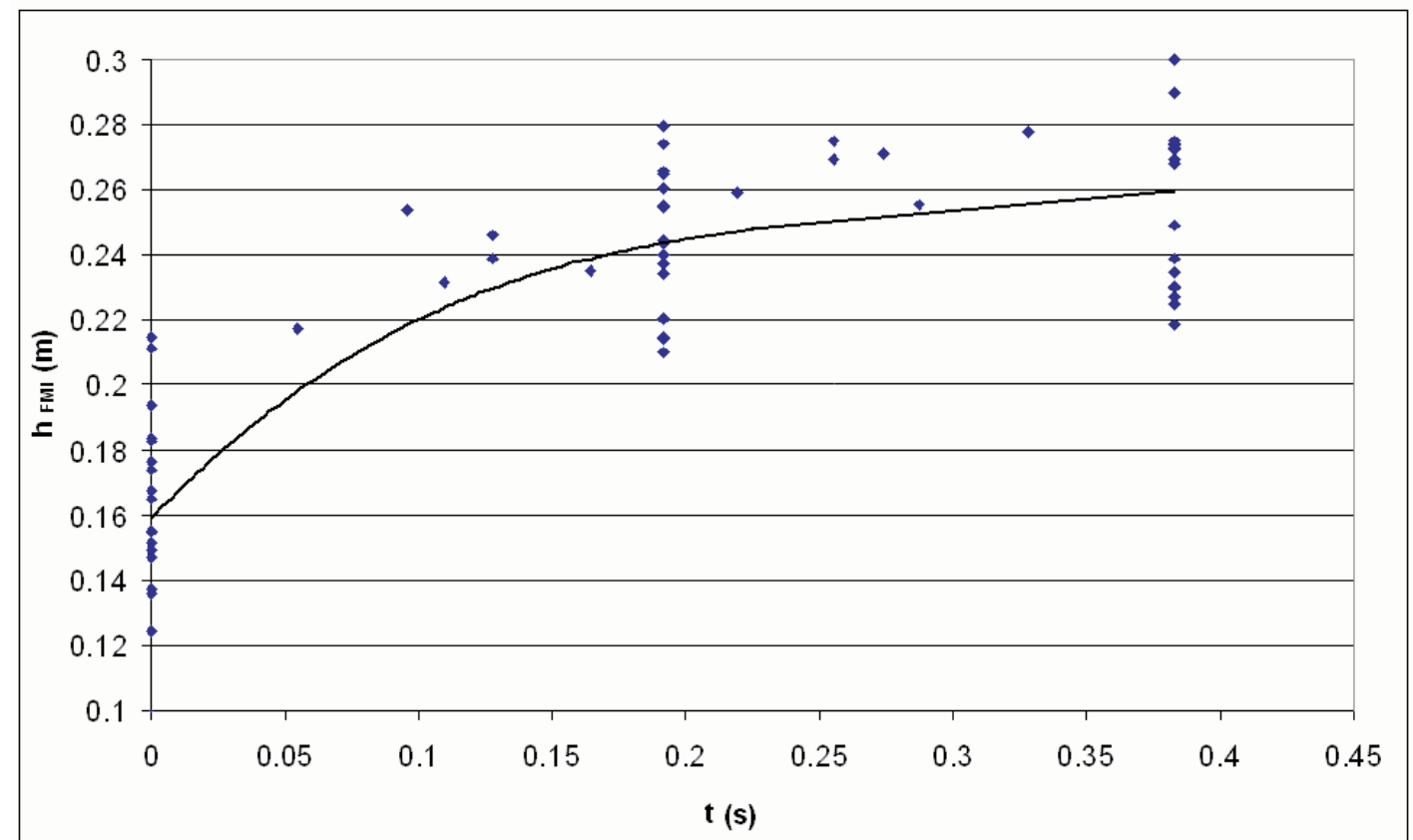

Fig. 10: Dependence of the height of the top of the flow macro formation $h_{\mathrm{FMI}, \mathrm{i}}$ on the time for one cycle

The calculated average time of one cycle (the time between the origin of the PCL and its collapse) is $t_{\mathrm{c}, \mathrm{av}}=1.59 \mathrm{~s}$. This means that the frequency of the PCL oscillations is $f_{\mathrm{osc}}=1 / t_{\mathrm{c}, \mathrm{av}}=0.63 \mathrm{~Hz}$ and dimensionless frequency $F_{\text {osc }}=f_{\text {osc }} / n=0.094$.

This dimensionless frequency is markedly higher than the value detected earlier in the interval 0.02-0.06 [8-10]. This corresponds to our finding that only some of the flow formations generated by one PCL cycle result in macro-flow formation causing a surface level eruption, detected as "macroinstability".

The function $h_{\mathrm{c}}=h_{\mathrm{c}}(t)$ for one cycle is shown in Fig. 8. This function was obtained by regression of the experimental data $h_{\mathrm{ci}}(t)$, where the individual cycle intervals were recalculated for an average time cycle $t_{\mathrm{c} \text {,av }}=1.59 \mathrm{~s}$. Fig. 8 shows that the mean maximum height of the PCL hc, max reaches a value of $0.181 \mathrm{~m}$, which is $62 \%$ of surface level height $H$. This agrees with earlier findings [11] that $h_{\mathrm{c}, \max } \approx 2 / 3 H$. The function $h_{\mathrm{c}}=h_{\mathrm{c}}(t)$ was used for calculating the PCL rising velocity, see Fig. 9.

Finally, the average rising time of flow macro formation generated by PCL was calculated from the time function $h_{\mathrm{FMI}}=h_{\mathrm{FMI}}(t)$ (time between generating and disintegration), and the value obtained was $t_{\mathrm{av}, \mathrm{FMI}}=1.0 \mathrm{~s}$.

The regression curve $h_{\mathrm{FMI}, \mathrm{i}}=h_{\mathrm{FMI}, \mathrm{i}}(t)$ corresponding to the experimental data for one cycle (development of one macro-formation, the time recalculated for average rising time $\left.t_{\mathrm{av}, \mathrm{FMI}}\right)$ is illustrated in Fig. 10.

To specify the PCL characteristics more deeply, the volume of the PCL (active volume of the primary circulation) was calculated. This was assumed as a toroidal volume around its elliptical projection in the $\mathrm{r}-\mathrm{z}$ plane of the mixing vessel. The function of the ratio of the PCL volume to vessel volume $V_{\mathrm{c}} / V=\left(V_{\mathrm{c}} / V\right)(t)$ for one cycle is illustrated in Fig. 11, where

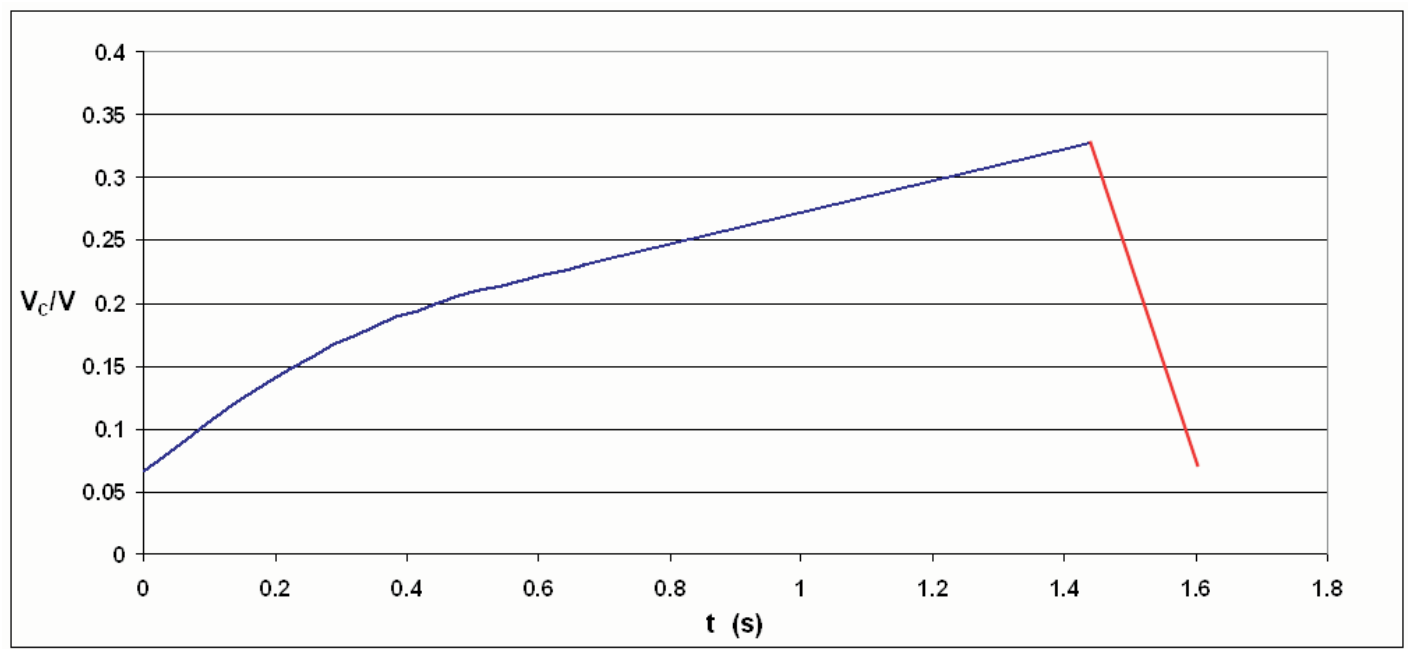

Fig. 11: Dependence of the ratio of the PCL volume to the vessel volume Vc,max/V on time for one cycle of the PCL oscillation 


$$
V_{\mathrm{c}}(t)=\frac{\pi^{2}}{2}\left(\frac{T h_{\mathrm{c}}(t) s_{\mathrm{c}}(t)}{4}-T r_{\mathrm{c}, \mathrm{av}}^{2}(t)\right)
$$

is calculated from the corresponding regression curves $h_{\mathrm{C}}=h_{\mathrm{c}}(t), s_{\mathrm{C}}=s_{\mathrm{C}}(t)$ and $r_{\mathrm{c}, \text { av }}(t)$. Fig. 11 shows that the mean maximum ratio of the PCL volume to the vessel volume $V_{\text {c,max }} / V$ reaches a value of 0.326 .

\section{Theoretical calculation of energy balance}

An energy balance for the PCL was carried out with a view to explaining the reasons for the flow field behaviour (predominantly PCL oscillations). As mentioned above, there are three different stages in the flow process. The energy balance was carried out for the quasi-steady stage under conditions when the PCL reaches its top position, i.e. $h_{\mathrm{c}}=h_{\mathrm{c} \text {, max }}$, see Fig. 12.

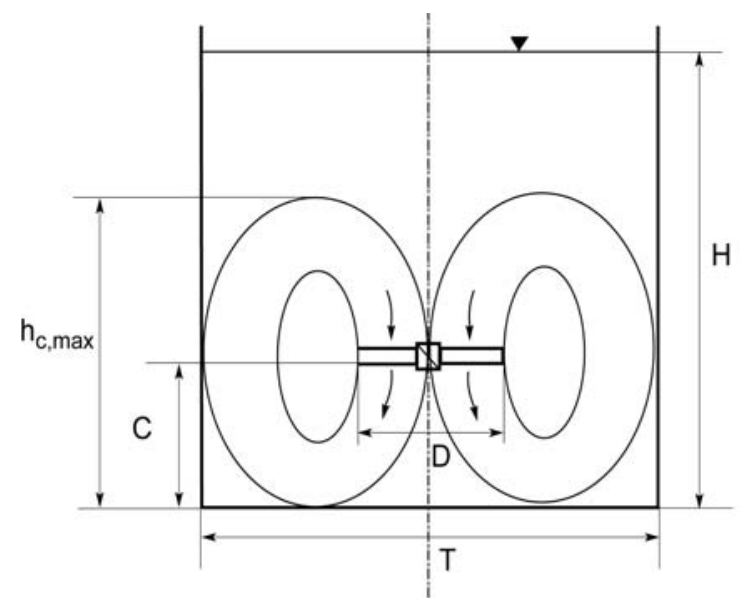

Fig. 12: Schematic view of the PCL under its top position $\left(h_{\mathrm{c}}=h_{\mathrm{c}, \max }\right)$

This stage directly follows (precedes) the collapse and is assumed to have a critical influence on the flow disintegration.

The impeller power input $N$ is dissipated in the PCL by the following components:

I) Mechanical energy losses:

1) $N_{\text {turn }}$ - lower turn of the primary circulation loop about $180^{\circ}$ from downwards to upwards.

2) $N_{\text {wall }}$ - friction of the primary circulation loop along the vessel wall.

II) Turbulence energy dissipation:

1) $N_{\text {disch }}$ - dissipation in the impeller discharge stream just below the impeller.

2) $N_{\text {up }}$-dissipation in the whole volume of the agitated liquid above the impeller rotor region, i.e. in the space occupied by both the primary and secondary flow.

It is expected that under quasi-steady state conditions (just before the PCL collapses) these components are in balance with the impeller power output. This means that no spare power is available, and that no alternative steady state formation is possible. The individual power components and quantities can be calculated when the validity of the following simplifying assumption for the flow in the PCL is considered:

1) The system is axially symmetrical around the axis of symmetry of the vessel and impeller.

2) The primary circulation loop can be considered as a closed circuit.

3) The cross section of the primary circulation loop (stream tube) is constant.

4) The conditions in the primary circulation loop are isobaric.

5) The liquid flow regime in the whole agitated system is fully turbulent.

6) The character of the turbulence in the space above the impeller rotor region is homogeneous and isotropic.

\subsection{Calculation of basic quantities}

Impeller power input

$$
P=P o \rho n^{3} D^{5},
$$

where the impeller power number $P o=1.7$ for $\operatorname{Re}_{\mathrm{M}}>10^{4}$ [12], $n=6.67 \mathrm{~s}^{-1}, D=0.0967 \mathrm{~m}, \rho=1000 \mathrm{~kg} / \mathrm{m}^{3}$. Then $P=4.25 \mathrm{~W}$.

The impeller power output can be calculated for known impeller hydraulic efficiency $\eta \mathrm{h}\left(\eta \mathrm{h}=0.48\right.$ for a four $45^{\circ}$ pitched blade impeller [14]).

$$
N=\eta_{h} P=2.04 \mathrm{~W} \text {. }
$$

Impeller pumping capacity $Q_{\mathrm{p}}$ can be calculated from the known flow rate number $N_{\mathrm{Qp}}\left(N_{\mathrm{Qp}}=0.94\right.$ for $\left.\operatorname{Re}_{\mathrm{M}}>10^{4}[13]\right)$.

$Q_{\mathrm{p}}=N_{\mathrm{Qp}} n D^{3}=5.66 \cdot 10^{-3} \mathrm{~m}^{3} \mathrm{~s}^{-1}=5.66 \mathrm{ls}^{-1}$

The mass flow rate is

$$
m_{\mathrm{p}}=Q_{\mathrm{p}} \rho=5.66 \mathrm{~kg} \mathrm{~s}^{-1}\left(\rho=1 \mathrm{~kg} \mathrm{l}^{-1}\right) .
$$

The axial impeller discharge velocity is equal to the average circulation velocity of the PCL (assuming a constant average circulation velocity in the loop):

$$
w_{\mathrm{c}, \mathrm{av}}=\frac{4 Q_{\mathrm{p}}}{\pi D^{2}}=0.77 \mathrm{~m} \mathrm{~s}^{-1},
$$

where $\pi \mathrm{D}^{2} / 4$ is the cross sectional area of the impeller rotor region, i.e. the cylinder circumscribed by the rotary mixer. Then the primary circulation loop is a closed stream tube consisting of the set of stream lines passing through the impeller rotor region.

\subsection{Calculation of turbulent dissipation below the impeller rotor region, $N_{\mathrm{disch}}$}

The energy dissipation rate per unit mass is [14]

$$
\varepsilon=A^{\prime} \frac{q^{3 / 2}}{L},
$$

where

$$
A^{\prime}=\left(\frac{2}{3}\right)^{3 / 2}
$$

and the integral length of turbulence [14] is

$$
L=\frac{D}{10} \text {. }
$$


The kinetic energy of turbulence per unit of mass is

$$
q=\frac{1}{2}\left(\overline{w_{z}^{\prime 2}}+\overline{w_{r}^{\prime 2}}+\overline{w_{\theta}^{\prime 2}}\right) .
$$

According to [14] the average value of $q$ in the impeller discharge stream is $q_{0}=0.226 \mathrm{~m}^{2} \mathrm{~s}^{-2}$ for the conditions described in [14]: a four pitched blade impeller with a pitch angle of $45^{\circ}, D_{0}=0.12 \mathrm{~m}, T_{0}=0.24 \mathrm{~m}, n_{0}=6.67 \mathrm{~s}^{-1}, P o_{0}=1.4$, $\operatorname{Re}_{\mathrm{M}, 0}=44 \cdot 10^{4}, P_{0}=10.3 \mathrm{~W}, L_{0}=0.012 \mathrm{~m}$. We have from Eq. (7) $\varepsilon_{0}=6.029 \mathrm{~m}^{2} \mathrm{~s}^{-3}$.

The energy dissipation per unit mass $\varepsilon$ related to the experimental system used in this study is

$$
\varepsilon=\varepsilon_{0} \frac{P / m}{P_{0} / m_{0}}=\varepsilon_{0} \frac{P / V}{P_{0} / V_{0}},\left(\rho=\rho_{0}\right)
$$

and after substitution $V=0.0192 \mathrm{~m}^{3}, P_{0}=10.3 \mathrm{~W}, V_{0}=0.0109$ $\mathrm{m}^{3}$ we obtain $\varepsilon=1.384 \mathrm{~m}^{2} \mathrm{~s}^{-3}$.

Using

$$
N_{\text {disch }}=\varepsilon \rho V_{\text {disch }},
$$

where the dissipation volume below the impeller rotor region $V_{\text {disch }}=0.000330 \mathrm{~m}^{3}$ (see Fig. 13). We obtain $N_{\text {disch }}=0.46 \mathrm{~W}$.

\subsection{Calculation of dissipation in the whole volume above the impeller region, $N_{u p}$}

According to [15]

$$
N_{\text {up }}=\eta_{\text {up }} P=0.60 \mathrm{~W} \text {, }
$$

where the portion of the impeller power input dissipated above the impeller rotor region $\eta_{\text {up }}=0.14$ for a six $45^{\circ}$ pitched blade impeller $(D / T=C / T=1 / 3, H=T)$ and $\operatorname{Re}_{\mathrm{M}}>10^{4}$.

When calculating the quantity $N_{\text {up }}$, the validity of introduced assumption No. 6 is considered. Moreover, because of the momentum transfer between the primary flow and the secondary flow out of the PCL the rate of energy dissipation above the impeller rotor region is related to the whole volume, consisting of both the primary and secondary flows.

\subsection{Calculation of the dissipation in the lower turns of the PCL, $N_{\text {turn }}$}

According to [16]

$$
N_{\text {turn }}=\sum_{j} \xi_{j} \frac{w_{\mathrm{c}, \mathrm{av}}^{2}}{2} m_{\mathrm{p}} .
$$

Substituting the sum of loss coefficients

$$
\sum_{j} \xi_{j}=0.50
$$

for bend of turn of the PCL $\beta=180^{\circ}$ as well as the values of the average circulation velocity of the PCL $w_{\mathrm{c}, \mathrm{av}}$, and the mass flow rate $m_{\mathrm{p}}$, we obtain $N_{\text {turn }}=0.84 \mathrm{~W}$.

\subsection{Calculation of the dissipation by the wall friction along the $P C L, N_{\text {wall }}$}

Using the relation for mechanical energy loss due to the friction along the wall, from [17]

$$
N_{\text {wall }}=\lambda \frac{l w_{\mathrm{c}, \mathrm{av}}^{2}}{d_{e}^{2}} m_{\mathrm{p}},
$$

where equivalent diameter is defined as

$$
d_{e}=\frac{4 S}{O}=\frac{\frac{4 \pi D^{2}}{4}}{\pi T}=\frac{D^{2}}{T}
$$

and the friction factor for a smooth wall

$$
\lambda=0.3164 \operatorname{Re}^{-0.25}
$$

where

$$
\operatorname{Re}=\frac{w_{\mathrm{c}, \mathrm{av}} D \rho}{\eta} .
$$

Then we can calculate the rate of energy dissipation in the flow along the smooth vessel wall after substitution: $\eta=1 \mathrm{mPa} \cdot \mathrm{s}$, length of the PCL along the wall $l=h_{\mathrm{c}, \max }-h_{\text {turn }} \quad$ where $h_{\mathrm{c}, \max }=0.181 \mathrm{~m}, \quad($ see Fig. 8), $h_{\text {turn }}=0.051 \mathrm{~m}$, so $l=0.13 \mathrm{~m}$, and values $d_{\mathrm{e}}=0.032 \mathrm{~m}$,

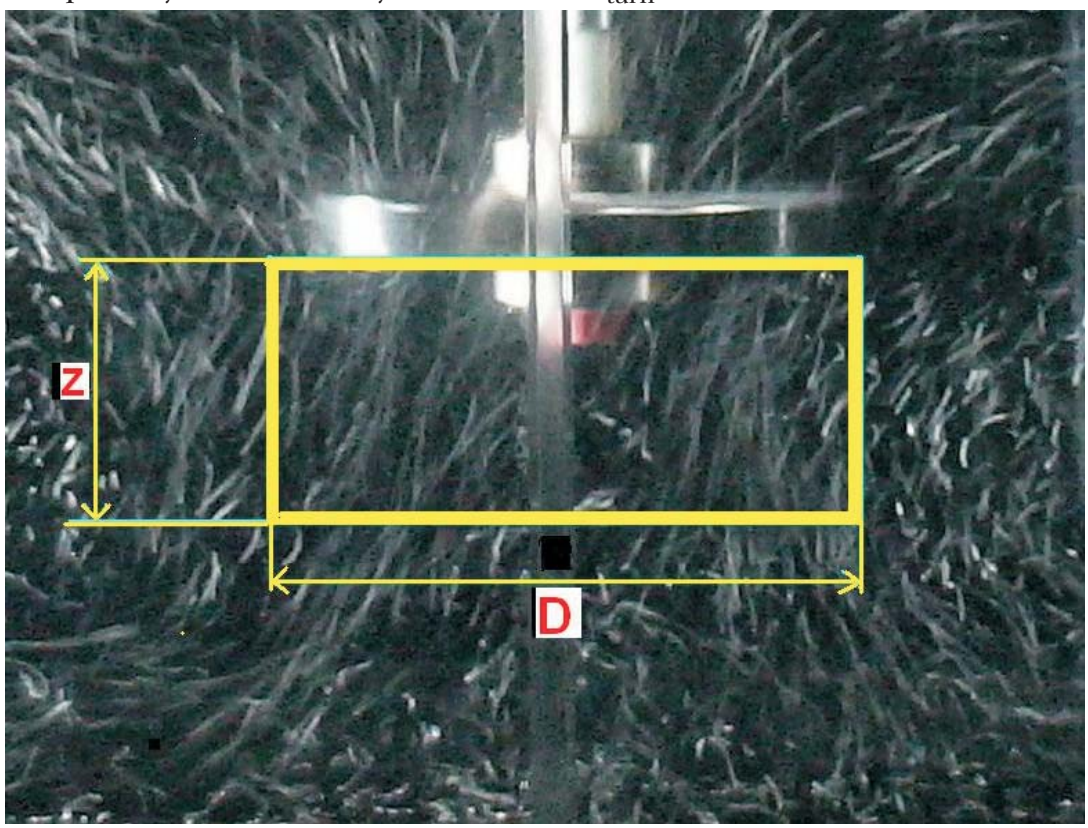

Fig. 13: Dissipation volume below the impeller rotor region $V_{\text {disc }}$ before the first turn of the loop $(D=T / 3=0.0967 \mathrm{~m}, z=0.045 \mathrm{~m})$ 
$\operatorname{Re}=7.45 \cdot 10^{4}$ and $\lambda=0.020$ obtained from Eqs. (16)-(18) to Eq. (15), we get $N_{\text {wall }}=0.14 \mathrm{~W}$.

\subsection{Energy balance in the quasi-steady stage of the PCL}

The sum of all dissipated power components considered here is

$\sum N_{i}=N_{\text {turn }}+N_{\text {wall }}+N_{\text {disch }}+N_{\text {up }}$,
$\sum N_{i}=0.84+0.14+0.46+0.60=2.04 \mathrm{~W}$.

The value in Eq. (19a) is in good agreement with the impeller power output from Eq. (3), $N=2.04 \mathrm{~W}$, though the data used for the calculations come from six independent literature sources. This result means that in the quasi-steady phase of the PCL oscillation cycle, all the impeller power output is consumed by dissipation. No power is available, either for increasing the PCL kinetic energy or for any changes in flow formation resulting in a higher energy level. However, it is well known that vortex disintegration in smaller formations or even a very small vortex separation from a primary flow is a process that consumes energy (according to the law of angular momentum conservation). The experiments proved that vortex separations (small and large) occur in all the stages, thus also in the quasi-steady stage. This seems to provide an explanation for the PCL collapse: in the quasi-steady stage, the energy necessary for vortex separation or for other changes in flow formation cannot be supplied by the impeller, but energy is exhausted from the ambient flow field. And then, even a very small energy deficit can result in a qualitative flow field change appearing as the PCL collapse. It should be noted that the PCL collapse can be followed by a noticeable rise in the surface level, classified as a macroinstability. However, not every collapse reaches the level and is observed as a macroinstability. This corresponds to this disagreement between the PCL oscillation frequency determined experimentally in this study and the macroinstability frequency presented.

The phenomenon observed here affects the processes taking place in an agitated charge, especially on the macro level, i.e., when miscible liquids blend and when solids suspend in liquids. Oscillations of the macroflow contribute to attain a so called "macroequilibrium" in an agitated batch, i.e., better homogeneity both in a pure liquid (blending) and in a solid-liquid suspension (distribution of solid particles in a liquid). These processes can be observed predominantly in the subregion of low liquid velocity above the impeller rotor region, corresponding to approx. One third of the volume of the agitated charge. Oscillations of the primary circulation of an agitated liquid can contribute to the forces affecting the body of the impeller as well as the mixing vessel and its internals. This low frequency phenomenon probably need not have fatal consequences, because the standard design of industrial mixing equipment should have sufficient margins for unexpected events during processes running in an industrial unit.

\section{Conclusions}

a) The average circulation velocity in the primary circulation loop is more than one order of magnitude higher than the rising velocity of the loop.

b) The frequency of the primary loop oscillations is about one order of magnitude lower than the revolution frequence of the impeller.

c) The top of the disintegration of the primary circulation loop is a birthplace of macroinstabilities in the region of secondary flow.

d) The primary circulation loop collapses owing to disequilibrium between the impeller power output and the rate of dissipation of the mechanical energy in the loop. A small change (e.g. a small turbulent vortex or a small increase in the primary circulation loop) can have a great effect.

\section{Acknowledgments}

The authors of this paper are grateful for financial support from the following Czech Grants Agencies:

1. Czech Grant Agency Grant No. 104/05/2500.

2. Czech Ministry of Education Grant No. 1P05 LA 250.

3. Czech Ministry of Education Grant No. MSM6046137306.

\section{List of symbols}

C

$d_{e}$

D

$f_{\text {osc }}$

$F_{\text {osc }}$

$h_{\mathrm{c}}$

$h_{\text {ci }}$

$h_{\text {c, } \max }$

$h_{\mathrm{c}, \min }$

$h_{\mathrm{FMI}}$

$h_{\mathrm{FMI}, \mathrm{i}}$

$h_{\text {turn }}$

$H$

$l$

$L$

$m$

$m_{\mathrm{p}}$

$n$

$N$

$N_{\text {disch }}$

$N_{\text {Qp }}$

$N_{\text {turn }}$

$N_{\text {up }}$ width of baffle, $m$

off-bottom clearance, $\mathrm{m}$

equivalent diameter, $\mathrm{m}$

impeller diameter, $\mathrm{m}$

frequency of PCL oscillations, $\mathrm{Hz}$

dimensionless frequency of oscillations

value of height of the PCL obtained by regression, $\mathrm{m}$

experimental value of height of the PCL, $m$

maximal height of the PCL, $m$

minimal height of the PCL, $\mathrm{m}$

value of height of the top of macro formation obtained by regression, $m$

experimental value of height of the top of the flow macro formation, $\mathrm{m}$

height of turn of the PCL, m

height of water level, $\mathrm{m}$

length of the PCL adjacent to wall, $\mathrm{m}$ integral length scale of turbulence, $m$ mass of liquid in a stirred tank, $\mathrm{kg}$ mass flow rate, $\mathrm{kg} \mathrm{s}^{-1}$

impeller speed, $\mathrm{s}^{-1}$

impeller power output, $\mathrm{W}$

turbulent dissipation below the impeller rotor region, $\mathrm{W}$

impeller pumping number

dissipation in lower turns of the PCL, W

dissipation in the whole volume above the impeller rotor region, $\mathrm{W}$ 
$N_{\text {wall }}$ dissipation by the wall friction of the PCL, W

$P \quad$ impeller power input, $\mathrm{W}$

Po impeller power number

$q$

kinetic energy of turbulence per unit of mass, $\mathrm{m}^{2} \mathrm{~s}^{-2}$

$Q_{\mathrm{p}} \quad$ impeller pumping capacity, $\mathrm{m}^{3} \mathrm{~s}^{-1}$

$r_{\mathrm{c} \text {,av }} \quad$ equivalent mean diameter value of the PCL core, obtained by regression, $\mathrm{m}$

$r_{\mathrm{c}, \mathrm{av} \text { i }} \quad$ experimental value of equivalent mean diameter of the PCL core, $m$

Re Reynolds number

$\mathrm{Re}_{\mathrm{M}} \quad$ impeller Reynolds number

$S \quad$ cross section, $\mathrm{m}^{2}$

$s_{\mathrm{C}} \quad$ value of width of the PCL, obtained by regression, $\mathrm{m}$

$s_{\mathrm{ci}}$

$T$

$t_{\mathrm{c}, \mathrm{av}}$

$t_{\mathrm{av}, \mathrm{FMI}}$

$u_{\mathrm{r}}$

$u_{\mathrm{r}, \mathrm{av}}$

V

$V_{\mathrm{c}}$

$V_{\text {c,max }}$

$V_{\text {disch }}$

W

$w_{\text {c,av }}$

$w_{\mathrm{z}}^{\prime}, w_{\mathrm{r}}^{\prime}, w_{\theta}^{\prime}$

$z$

$\beta$

$\varepsilon$

$\eta$

$\eta_{\mathrm{h}}$

$\eta_{\text {up }}$

$\lambda$

$\rho$

$\zeta_{j}$

\section{References}

[1] Roussinova, V. T., Kresta, S. M., Weetman, R.: Low Frequency Macroinstabilities in Stirred Tank: Scale-up and
Prediction Based on Large Eddy Simulations. Chemical Engineering Science., Vol. 58 (2003), p. 2297-2311.

[2] Roussinova, V. T., Kresta, S. M., Weetman, R.: Resonant Geometries for Circulation Pattern Macroinstabilities in a Stirred Tank. AIChE Journal, Vol. 50-12 (2004), p. 2986-3005.

[3] Roussinova, V. T., Grcic, B., Kresta, S. M.: Study of Macro-Instabilities in Stirred Tanks Using a Velocity Decomposition Technique. Trans I ChemE, Vol. 78A (2000), p. 1040-1052.

[4] Fan, J., Rao, Q., Wang, Y., Fei, W.: Spatio-Temporal Analysis of Macro-instability in Stirred Vessel via Digital Particle Image Velocimetry (DPIV). Chemical Engineering Science, Vol. 59 (2004), p. 1863-1873.

[5] Ducci, A., Yianneskis, M.: Vortex Tracking and Mixing Enhancement in Stirred Processes, AIChE Journal, Vol. 53 (2007), p. 305-315.

[6] Fořt, I., Gračková, Z., Koza, V.: Flow Pattern in a System With Axial Mixer and Radial Baffles. Collection of Czechoslovak Chemical Communications, Vol. 37 (1972), p. 2371-2385.

[7] Kresta, S. M., Wood, P. E.: The Mean Flow Field Produced by a $45^{\circ}$ Pitched Blade Turbine: Changes in the Circulation pattern Due to off Bottom Clearance. The Canadian Journal of Chemical Engineering, Vol. 71 (1993), p. 52-42.

[8] Brůha, O., Fořt, I., Smolka, P.: Phenomenon of Turbulent Macro-Instabilities in Agitated Systems. Collection of Czechoslovak Chemical Communications, Vol. 60 (1995), p. 85-94.

[9] Hasal, P., Montes, J-L., Boisson, H. C., Fořt, I.: Macro-instabilites of Velocity Field in Stirred Vessel: Detection and Analysis. Chemical Engineering Science, Vol. 55 (2000), p. 391-401.

[10] Paglianti, A., Montante, G., Magelli, F.: Novel Experiments and Mechanistic Model for Macroinstabilities in Stirred Tanks. AICHE Journal, Vol. 52 (2006), p. $426-437$.

[11] Bittorf, K. V., Kresta, S. M.: Active Volume of Mean Circulation for Stirred Tanks Agitated with Axial Impellers. Chemical Engineering Science, Vol. 55 (2000), p. 1325-1335.

[12] Medek, J.: Power Characteristics of Agitators with Flat Inclined Blades. International Chemical Engineering, Vol. 20 (1985), p. 664-672.

[13] Brůha, O., Fořt, I., Smolka, P., Jahoda, M.: Experimental Study of Turbulent Macroinstabilities in an Agitated System with Axial High-speed Impeller and Radial Baffles. Collection of Czechoslovak Chemical Communications, Vol. 61 (1996), p. 856-867.

[14] Zhou, G., Kresta, S. M.: Distribution of Energy Between Convective and Turbulent Flow for Three Frequently Used Impellers. Trans I ChemE, Vol. 74 (Part A) (1996), p. 379-389.

[15] Jaworski, Z., Fořt, I.: Energy Dissipation Rate in a Baffled Vessel with Pitched Blade Turbine Impeller. Collection of Czechoslovak Chemical Communications, Vol. 56 (1991), p. 1856-1867. 
[16] Perry, J. H.: Chemical Engineer's Handbook (Fourth Edition). New York: McGraw-Hill Book Comp., 1963.

[17] Brodkey, R. S.: The Phenomenon of Fluid Motions. Reading: Adison-Wesley Publishing Comp., 1967.

Doc. Ing. Oldřich Brůha, CSc. phone, fax: +420251560040 mobil: +420777 895766

e-mail: enex@atlas.cz

Department of Physics

Czech Technical University in Prague

Faculty of Mechanical Engineering

Technická 4

16607 Prague 6, Czech Republic

Ing. Tomáš Brůha

phone, fax: +420251560040

mobil: +420777895762

e-mail: enex@volny.cz

Department of Chemical and Process Engineering
Institute of Chemical Technology, Prague

Technická 5

16628 Prague 6, Czech Republic

Doc. Ing. Ivan Fořt, DrSc.

phone: +420224352713

fax: +420224310292

e-mail: Ivan.Fořt@fs.cvut.cz

Department of Process Engineering

Czech Technical University in Prague

Faculty of Mechanical Engineering

Technická 4

16607 Prague 6, Czech Republic

Doc. Dr. Ing. Milan Jahoda

phone: +420220443223

fax: +420220444320

e-mail: Milan.Jahoda@vscht.cz

Department of Chemical and Process Engineering

Institute of Chemical Technology, Prague

Technická 5

16628 Prague 6, Czech Republic 